\title{
pnrVSA: human-like actuator with non-linear springs in agonist-antagonist configuration
}

\author{
Luca Fiorio, Alberto Parmiggiani, Bastien Berret, Giulio Sandini and Francesco Nori \\ Department of Robotics, Brain and Cognitive Sciences \\ Istituto Italiano di Tecnologia, Via Morego 30, Genova, ITALY \\ Email: \{luca.fiorio, alberto.parmiggiani, bastien.berret, giulio.sandini, francesco.nori\}@iit.it
}

\begin{abstract}
One of the most important features for a system capable of working in uncertain and unstructured environments is reliability. Nowadays robots are excellent machines, but are still not able to interact with their surrounding environment as humans or animals do. Recent studies highlight the role played by impedance changes in the human arm during manipulation tasks. In particular the possibility to vary the stiffness of shoulder, elbow and wrist allows humans to interact easily with fast changing environments while rejecting unpredictable noise disturbances [1]. Several studies also showed how the capability of "co-contracting" antagonistic muscles is required to interact with noisy/unpredictable environments. Starting from these premises we recently proposed novel design principles to build actuators with the ability to actively regulate the passive noise rejection (i.e. the ability to cancel the effect of disturbances without explicitly relying on feedback) [2]. In the present paper we implement these principles in the mechanical design of a novel actuator. The actuator is composed of two electric motors in agonist-antagonist configuration. The final design includes also four non-linear springs whose force-displacement characteristic has been customized on the specific application requirements. Validation of the proposed non-linear spring design has been conducted on a prototype and results are reported in this paper. Future works foreseen the integration of the proposed actuators on a two limbed robot with six artificial muscle, (three agonist-antagonist pairs) in a simple and biarticular configuration.
\end{abstract}

\section{INTRODUCTION}

In the last decade, roboticisits have started studying mechanical solutions capable of controlling the system structural stiffness by proposing a number of solutions [3], [4] which fall under the broad category of passive Variable Stiffness Actuators (pVSA). All these systems use different principles (cam mechanisms, nonlinear springs, etc.) to change the rigidity between actuator and joint in order to mimic human ability to change the body compliance by regulating the muscle co-activation. Although the proposed designs embed interesting features, we recently pointed out [5] that available solutions strongly rely on feedback control strategies and differ (as to this concern) from human muscles. Specifically simulations indicated that muscle models outperform available pVSA solutions in dealing with unpredictable (i.e. stochastic) perturbations [5]. These results motivated us to design a different type of pVSA possessing a novel property that we call passive noise rejection (pnrVSA). The design was inspired by recent motor control experiments showing that humans adopt muscle co-activation as a strategy to deal with highly unstable force fields in presence of

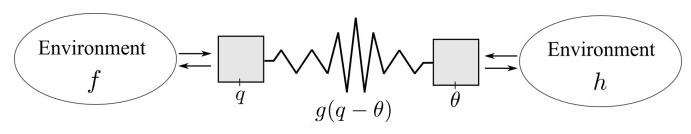

Fig. 1. A sketch of VSA joints represented for sake of simplicity as a linear actuator. The motor shaft (whose position is indicated with $\theta$ ) is coupled to the joint (whose position is indicated with $q$ ) by a variable stiffness spring (indicated with $g$ ). The force acting on the motor shaft is the system input, therefore indicated with $u$. All other forces acting on the joint and on the motor shaft are indicated with the functions $f$ and $h$ respectively.

significant proprioceptive delays [1]. In order to reproduce a similar capability in robots, we propose the pnrVSA design with the final goal of building an artificial arm able to execute intrinsically unstable tasks without explicitly relying on feedback.

The paper is organized as follows. Section II proposes a summary of the mathematical framework which guided the design of the proposed pnrVSA ${ }^{1}$. Section III discusses the final design choice together with the associated mechanical properties. Section IV presents the single joint actuator together with the procedure adopted to realize non-linear springs with custom force-displacement characteristic. Finally, Section IV-B validates the design procedure presenting the results obtained on two non-linear spring prototype.

\section{PNRVSA DESIGN PRINCIPLE}

In this section we consider a broad class of pVSA and we compute the associated passive disturbance rejection. The proposed analysis gives us multiple design choices to realize the desired innovative property, nominally the capability of actively mimic the human co-contraction augmenting the passive disturbance rejection [1]. Among these multiple design choices, one specific is chosen and described in details in the following section.

The key component of a typical pVSA is an elastic element of variable stiffness in between the motor shaft (whose position will be indicated with $\theta$ and generated force will be indicated with $u$ ) and the joint (indicated with $q$ ). In Fig. 1 this elastic element is represented with a function $g$. The

\footnotetext{
${ }^{1} \mathrm{~A}$ more detailed analysis of these design principles is the content of the submitted paper "Design principles for muscle-like variable impedance actuators with noise rejection property via co-contraction".
} 
associated dynamic equation is the following:

$$
\left\{\begin{array}{l}
\ddot{q}=f(q, \dot{q})+g(q-\theta)+u \\
\ddot{\theta}=h(\theta, \dot{\theta})-g(q-\theta)
\end{array} .\right.
$$

When linearized around an equilibrium configuration $(q=$ $\left.q_{e q}, \theta=\theta_{e q}\right)$ the system dynamics become:

$\dot{x}=\left(\begin{array}{cccc}0 & 0 & 1 & 0 \\ 0 & 0 & 0 & 1 \\ a+c & -c & d & 0 \\ -c & b+c & 0 & e\end{array}\right) x+\left(\begin{array}{l}0 \\ 0 \\ 1 \\ 0\end{array}\right) u, \quad x=\left(\begin{array}{c}q-q_{e q} \\ \theta-\theta_{e q} \\ \dot{q}-\dot{q}_{e q} \\ \dot{\theta}-\dot{\theta}_{e q}\end{array}\right)$

where the state $x$ represents deviations from the equilibrium configuration and $a, \ldots, e$ have the following meaning:

$$
a=\frac{\partial f}{\partial q}, b=\frac{\partial h}{\partial \theta}, c=\frac{\partial g}{\partial q}, d=\frac{\partial f}{\partial \dot{q}}, e=\frac{\partial h}{\partial \dot{\theta}}
$$

with derivatives all computed at the equilibrium configuration. The model that we just obtained represents the (linearized) dynamic equation of a pVSA. We now study the effect of disturbances entering the system. As it was previously pointed out, we are mainly interested in the passive disturbance rejection, i.e. we are not resorting to feedback to increase disturbance rejection. Disturbance will be represented by stochastic variables acting as forces on the motor shaft (white noise of variance $\sigma_{\theta}$ ) and on the joint (white noise of variance $\sigma_{q}$ ). A quantitative measure of disturbance rejection is represented by the covariance matrix $P$, defined as the steady-state covariance of the state vector $x$ in response to the applied stochastic perturbations, i.e. $P=$ $\lim _{t \rightarrow \infty} E\left[(x-E[x])(x-E[x])^{\top}\right]$ being $E[\cdot]$ the expected value of a stochastic variable. The smaller the eigenvalues of the matrix $P$, the higher the passive disturbance rejection. In particular, a good quantitative measurement for the passive disturbance rejection is the trace of the matrix $P$, corresponding to the sum of the eigenvalues. Computations not reported here can produce an analytical expression for the matrix $P$ (which results from the solution of an associated Lyapunov equation). Given the complexity of this analytical expression, we report here some simplified equations, which are the limits as $c$ approaches its extremes (the focus on $c$ being motivated by the fact that available pVSA are already capable of changing its value). We have:

$$
\begin{array}{r}
\lim _{c \rightarrow 0} \operatorname{trace}(P)=\frac{1}{2} \frac{\sigma_{q} b e(a+1)+\sigma_{\theta} a d(a+1)}{a b d e}, \\
\lim _{c \rightarrow \infty} \operatorname{trace}(P)=\frac{(a+b)\left(\sigma_{q}^{2}+\sigma_{\theta}^{2}\right)+\left(\sigma_{q}+\sigma_{\theta}\right)^{2}}{(a+b)(d+e)}, \\
\left.\frac{\partial \operatorname{trace}(P)}{\partial c}\right|_{d=e=1}=\frac{1}{2} \frac{\left(a \sigma_{\theta}-b \sigma_{q}\right)^{2}}{(a c+b c+a b)^{2}} .
\end{array}
$$

Remarkably what these expressions show is that the passive disturbance rejection monotonically increases with the stiffness of the elastic element in between the motor and the actuator (represented by the parameter $c$ ). However, the values of $a, b, d$ and $e$ are also essential to guarantee a certain level of passive disturbance rejection. The mechanical meaning of these parameters is represented in Fig. 2. In practice they correspond to a linearization of the interaction

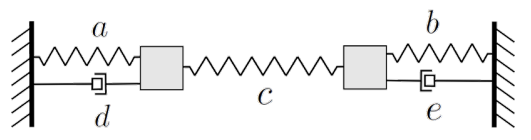

Fig. 2. The picture shows a schematic representation of the linearization of the system in Fig. 1. The elastic and damping elements $a$ and $b, c$ and $d$ correspond to the linearization of the environmental interactions with the joint and the motor respectively.

with the environment. The spring elements connecting the motor and the joint to the environment, $a$ and $b$ respectively, are typically not present in pVSA designs with rotary motors. Nevertheless, their crucial role in determining the overall system passive disturbance rejection, is evident in the formulas above, which diverge when $a$ and $b$ simultaneously tend to zero. In practice when $a=b=0$ the system is free-floating with respect to the environment and noise can drive the system arbitrarily far from the initial configuration. Therefore, we concentrate on actuators like the ones in Fig. 1, whose linearization (Fig. 2) do not have $a$ and $b$ simultaneously zero. We name these actuators "passive noise rejecting VSA" (pnrVSA), since the requirement $a \neq 0$ or $b \neq 0$ is a necessary condition for the system to passively reject disturbances.

So far, we only characterized the passive disturbance rejection properties of a quite general actuator class (Fig. 1). In the following, we describe how the passive disturbance rejection can be actively controlled. As it was previously discussed, the control of $c$ (the stiffness of the spring in between the actuator and the joint) already gives control on the passive disturbance rejection if either $a \neq 0$ or $b \neq 0$. However, all the other parameters $a, b, d$ and $e$ have an active role. If we are interested in maximizing the passive disturbance rejection (i.e. minimizing the trace of $P)$, these parameters play an important role since rejection is maximum if their values tend to infinity. In a sense passive noise rejection is increased by augmenting the stiffness of the path which connect the joint to the ground.

The implementation of this idea can be achieved in different ways; in this paper we follow a design based on nonlinear springs which can vary their equilibrium configuration thanks to an agonist-antagonist arrangement. The complete mechanical design is described in the following section, where details on a specific pnrVSA implementation are given. The actuator structure (schematized in Fig. 3) consists of an agonist-antagonist configuration which resembles the human muscles arrangement. All springs connecting the joint to the actuators and the actuators to the ground are non-linear (as indicated by the sketch with coils of variable width). The agonist-antagonist structure is such that co-activation of motors stretches the springs, changing their local stiffness therefore modifying the passive disturbance rejection at the joint. 


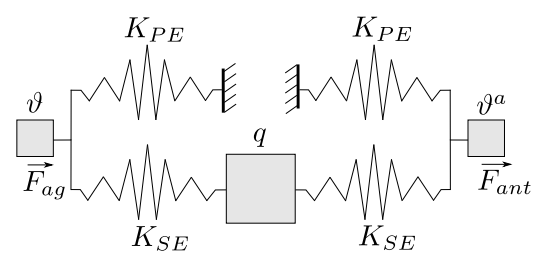

Fig. 3. The picture shows an agonist antagonist implementation of the pnrVSA actuator. All springs are non-linear, as indicated by the spring coils of variable width.

\section{PNRVSA MECHANISM CONCEPT}

In this section we propose the mechanical implementation of the actuator noise rejecting characteristic presenting also the complete planar manipulator. As highlighted in the previous section the main feature of our system is the possibility of finding a path that connects together joint, motor reel and frame and that can be stiffened. The principle sketched in Fig. 3 has been implemented with rotational motors which are more commonly used in the foreseen applications. The mechanical design shown in Fig. 4 is obtained by attaching two nonlinear springs on the opposite side of the motor reels: the first, named $K_{P E}$, realizes the connection to the ground and works in parallel with respect to the contractile element $\vartheta$ (spring elongation equals reel displacement); the second, named $K_{S E}$, behaves as series elastic element. Intuitively, a clockwise rotation of the motor $\vartheta$ coupled with a counterclockwise rotation of $\vartheta^{a}$ stretches all springs causing no movement of the joint $q$. If springs are designed in such a way that stiffness increases with stretch, the overall path connecting the joint to the ground is stiffened up, resulting in increased noise rejection. Similarly, rotating the two motors in the same clockwise direction intuitively results into a pure movement of the joint with no stiffness variation. A complete analysis and characterization of these intuitive control laws can be found in [2].

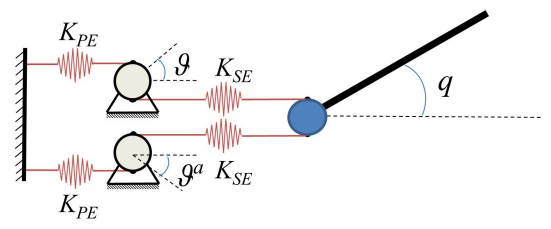

Fig. 4. The picture shows an agonist antagonist preliminary design of the actuator

Let's now discuss the relationship between the torques applied at the agonist $\left(\tau_{a g}\right)$ and antagonist $\left(\tau_{a n t}\right)$ motors and the corresponding forces $\left(F_{a g}, F_{a n t}\right)$ generated at the joint $q$. Generally speaking, motor torques contribute to stretching both $K_{P E}$ and $K_{S E}$, but only the second (i.e. stretching $K_{S E}$ ) produces forces at the joint. Applying equal and opposite internal torques $\left(\tau_{a g}=-\tau_{a n t}\right)$ results in equal and opposite angular displacement $\vartheta$ and $\vartheta^{a}$. With no external torques applied on $q$, forces contribution $\left(F_{a g}, F_{a n t}\right)$ on the joint $q$ are equal and opposite $\left(F_{a g}=-F_{a n t}\right)$. If an external torque is applied to $q$, the joint moves and consequently one artificial muscle is compressed while the other is stretched. In this case the forces due to the agonist and antagonist muscles differ, and the difference equals exactly the applied external torque. The muscle which is stretched, will contribute with more force on the joint as a consequence of the fact that stretching the muscle results in compressing $K_{P E}$ (see the sketch in Fig. 4) thus unbalancing in favor of $K_{S E}$ the percentage of motor torque contributing to the springs elongation. As a result, the stretched muscle can contributes with more force on the joint. Similarly, the compressed muscle contributes with less force on the joint. Qualitatively this analysis shows that the agonist/antagonist muscles act on $q$ as a restoring springs whose force curves $\left(F_{a g}\right.$ and $\left.F_{\text {ant }}\right)$ are shaped by the nonlinearity of $K_{P E}$. Quantitatively, the described actuator has been characterized computing the data sheet specifications (see Fig. 10) in agreement with the procedure formally described within the EU project Viactors. Springs characteristics are the one that will be described in Fig. 9.

The proposed actuator design has been optimized having in mind the application sketched in Fig. 5, a two degrees of freedom planar arm actuated by three of the proposed actuators. The system is composed by two limbs controlled by six motors connected to frame and joint with twelve nonlinear springs. In particular motors $M 1$ and $M 2$ control the first joint $q 1$, while motors $M 3, M 4, M 5$ and $M 6$ control the second joint $q 2$. In this case $M 5$ and $M 6$ are connected between main frame and the second limb in a biarticular configuration. The idea behind this design choice is the possibility of simultaneously controlling the orientation and shape of ellipse which represents the stiffness at the distal limb.

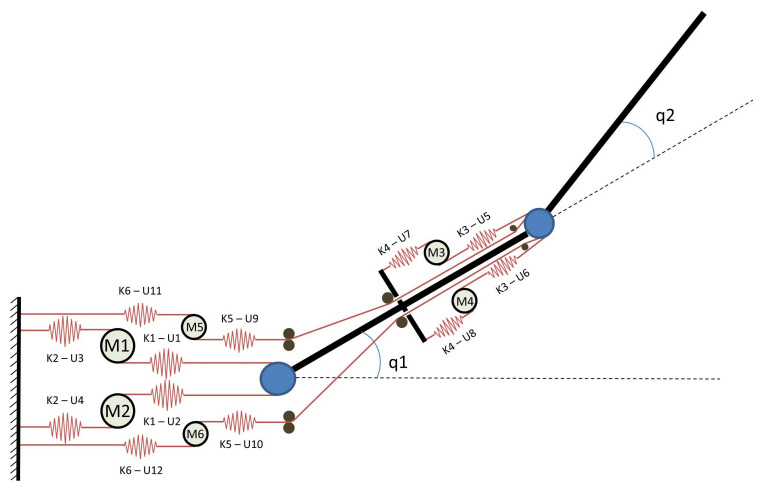

Fig. 5. pnrVSA - Manipulator scheme

As summarized in Tab. I the main required features of the system concern limb range of motion and resting position (that have been chosen to be equal humans arm joints [6], [7]) and limbs lengths (matching the iCub arm [8]). Only the range of motion of joint $q 1$ has been reduced to facilitate actuator design.

The choice of the six electric motors size, gearbox ratios and springs coefficients has been conducted using an optimization algorithm for each artificial muscle couple. In 


\begin{tabular}{ll}
\hline Properties & Values \\
\hline Time to reach maximum stiffness(s) & 0.2 \\
Joint 1 RoM(deg) & $-110 /+30$ \\
Joint 2 RoM(deg) & $0 /+120$ \\
Joint 1 neutral angle(deg) & -40 \\
Joint 2 neutral angle(deg) & 60 \\
Limb 1 length $(\mathrm{m})$ & 0.16 \\
Limb 2 length $(\mathrm{m})$ & 0.15 \\
Maximum weight $(\mathrm{Kg})$ & 4
\end{tabular}

TABLE I

PNRVSA - MANIPULATOR FEATURES

particular the following parameters have been considered:

- Minimum torque required at joint: $7 \mathrm{Nm}$

- Maximum transitory to reach highest stiffness : $0.2 \mathrm{~s}$

- Spring elongation range: $20-30 \mathrm{~mm}$

- Overshooting absence

- Motor reel radius: $0.0075 \mathrm{~m}$

- Joint 1 pulley radius: $0.025 \mathrm{~m}$

- Joint 2 pulley radius: $0.02 \mathrm{~m}$

The optimization process minimized a fitness function constructed by a simplified Simulink actuator model and an evaluation algorithm that weights the considered parameters. As explained in Section IV spring characteristic has been chosen to be quadratic. Furthermore as joint $q 2$ is controlled by four motors the torque has been split trying to have smaller hardware on-board proximal limb. Regarding the electronics, each couple of motors is controlled by BLLBLC board package [9]. The sensing system includes one incremental encoder for every motor (Faulhaber IE2-512) and fourteen absolute magnetic rotary encoders: one for each joint and one mounted inside all springs. Furthermore two six-axes force-torque sensors have been embedded in the two limbs to measure external forces.

\section{FOREARM AND SPRINGS MECHANICAL DESIGN}

In this section we present the mechanical design of the proximal limb consisting in one complete antagonist actuator focusing on the nonlinear spring selection, design and tests. Since joint stiffness variation is the effect of springs nonlinearity, the choice of the elastic elements heavily affects the performance of the system. In particular, as the following equations show, in agonist-antagonist systems there is an advantage by using quadratic springs because they allow a complete control of the stiffness. Eq. 2 express the generic quadratic relation between the nonlinear force $F(x)$ and the spring elongation $x$ :

$$
F(x)=K_{2} x^{2}+K_{1} x
$$

Can be easily obtained the following equilibrium equation for the torque $\tau$ at joint $q$ :

$$
\tau=-R r\left(\vartheta-\vartheta^{a}\right)\left(K_{1}+2 K_{2} R q-K_{2} \vartheta r-K_{2} \vartheta^{a} r\right)
$$

where $r$ is the radius of motor's reels, $R$ the radius of joint's pulleys, $\vartheta$ the angular position of agonist motors, $\vartheta^{a}$ the angular position of antagonist motors and $q$ the joint angular position. Differentiating with respect to $q$ it is possible to evaluate the joint stiffness:

$$
\frac{d \tau}{d q}=-2 K_{2} R^{2} r\left(\vartheta-\vartheta^{a}\right) .
$$

As mentioned before, the joint position is a linear function of the motor reel angular displacement. From Eq. 3 it is possible to derive the equilibrium position for $q$ when $\tau=0$ :

$$
q=-\frac{K_{2} \vartheta r-K_{1}+K_{2} \vartheta^{a} r}{2 K_{2} R} .
$$

Due to this constraint the nonlinear spring design was finalized to obtain a device able to show a predetermined nonlinear quadratic force-displacement law. Details on how to compute the system stiffness and equilibrium configuration with generic non-quadratic springs can be found in [2].

\section{A. Nonlinear spring design}

The nonlinear spring design has been optimized and customized in order to have light and compact solutions for both the parallel elastic element $K_{P E}$ and the series elastic element $K_{S E}$. Starting from the example reported in [10] we exploited the idea of "non-circular spool", where the change in stiffness is achieved through a cam varying radius, specialized into two different custom solutions. Regarding the $K_{S E}$ spring realization we have chosen a solution which connects a non-circular spool in parallel to a linear torsional spring as shown in the left hand side of Fig. 6. For the parallel elastic element $K_{P E}$ we had to consider that due to the wide range of motion of the output limb, this spool performs more than one rotation and has been therefore realized with a threedimensional cut of the non-circular profile (right hand side of Fig. 6). Furthermore in order to reduce the total spring length the design was optimized directly connecting the motor shaft to the spool, along whose axis a cable is winded up on a groove of variable depth (see again Fig. 6). This solution ensures the correct radius/angular displacement gain winding the cable connected to a linear compression spring. The design has been conducted with a slight modification of the procedure in [10].
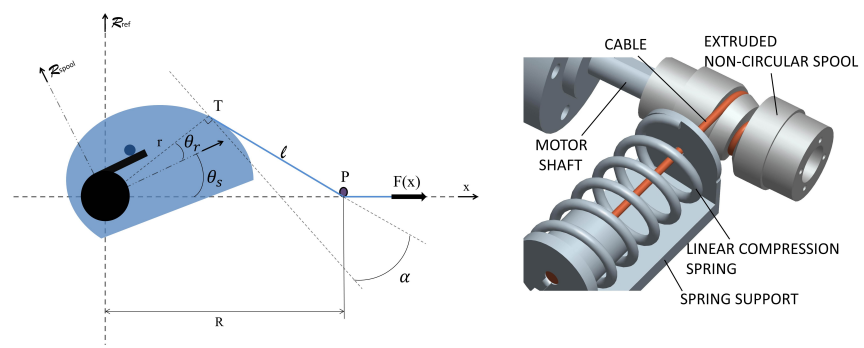

Fig. 6. The nonlinear springs. On the left side the scheme that represents the principle used to derive the $K_{S E}$ elastic element, while on the right side a schematic representation of the $K_{P E}$ elastic element.

The complete forearm is shown in Fig.7. The two electric motors are connected to the reels, that wind the cable acting 
on the $K_{S E}$ springs, and to the extruded non-circular spools that realize the parallel elastic elements $K_{P E}$.

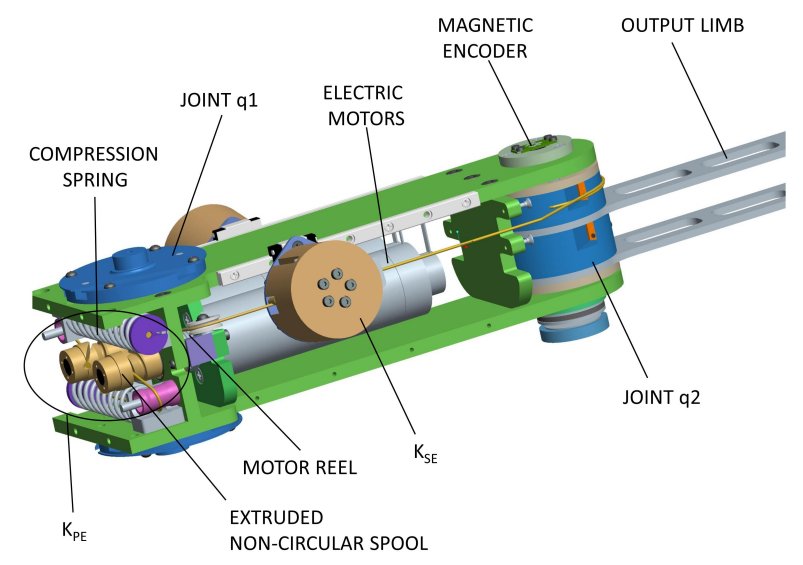

Fig. 7. pnrVSA - Complete forearm

\section{B. Nonlinear spring test}

In order to evaluate the performance of the proximal $\left(K_{P E}\right)$ and distal $\left(K_{S E}\right)$ springs we constructed and tested two prototypes with a Zwick Roell ProLine traction testing machine. Starting from the desired nonlinear forceelongation laws it has been possible to compute the profiles used to model the non-circular spools. Custom parts have been printed with a "Connex 500" 3D Printer employing the "ABS-like" Digital Material (RGD5160-DM). In Fig. 8 is shown the CAD view of the complete $K_{S E}$.

As shown in Fig. 9, the comparison of the theoretical (red) and experimental (blue) data gives good results with small errors.

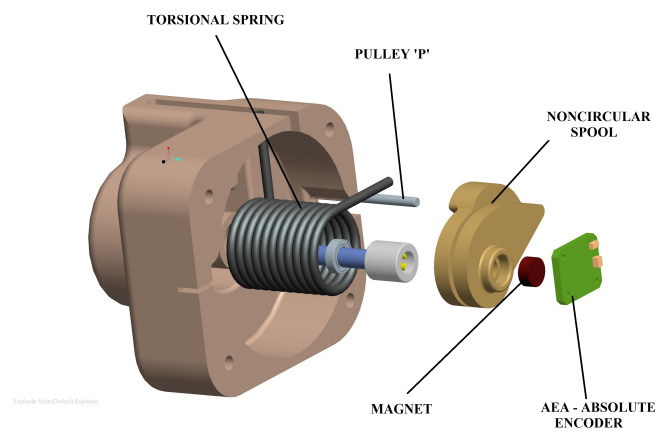

Fig. 8. Exploded view of nonlinear spring assemble

\section{CONCLUSION}

In this paper we presented the mechanical design of a novel actuator (pnrVSA) capable of actively changing its passive noise rejection characteristic. The proposed actuator is composed of two independent motors in an agonistantagonist configuration. Crucial elements in the proposed
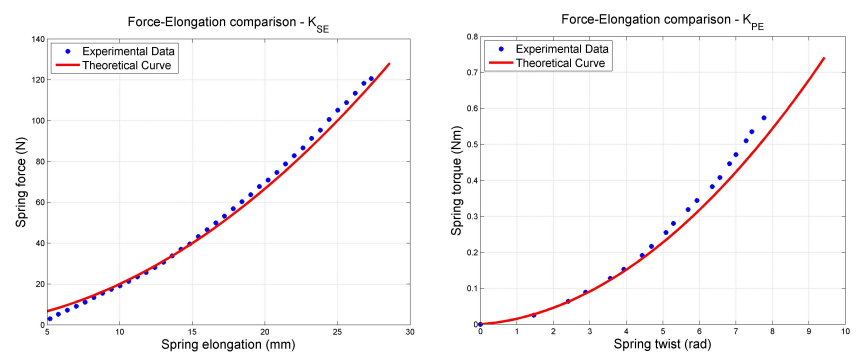

Fig. 9. These plots show the comparison between experimental result and theoretical curve. On the left side the $K_{S E}$ elastic element (Mean Square Error: $11.37[\mathrm{~N}]$ ), while on the right side the $K_{P E}$ elastic element (Mean Square Error: $0.0013[\mathrm{Nm}])$.

system are four non-linear springs whose force-displacement characteristic has been customized on the specific needs of the foreseen applications. The final solution adopts quadratic non-linear springs which have been build and tested to verify the validity of the approach. Future works include the realization of a two degrees of freedom robotic arm actuated by three of these actuators: two acting on a single joint, one spanning two joints in a polyarticular-like configuration.

\section{ACKNOWLEDGMENTS}

We would like to thank Lorenzo Orciari, Roberto Puddu, Mattia Salvi and Marco Randazzo whose help has been essential to the completion of this work. Emiliano Barbieri, Andrea Menini and Fabrizio Larosa for the support on the logistic side.

\section{REFERENCES}

[1] D. W. Franklin, G. Liaw, T. E. Milner, R. Osu, E. Burdet, and M. Kawato, "Endpoint stiffness of the arm is directionally tuned to instability in the environment," Journal of Neuroscience, 2007.

[2] F. Nori, B. Berret, L. Fiorio, A. Parmiggiani, and G. Sandini, "Control of a single degree of freedom noise rejecting-variable impedance," in Proceedings of the 10th international IFAC symposium on Robot Control (SYROCO2012), 2012.

[3] R. V. Ham, T. G. Sugar, B. Vanderborght, and D. Lefeber, "Compliant actuator design," Robotics \& Automation Magazine, IEEE, pp. 16 8194, 2009.

[4] A. Jafari, N. G. Tsagarakis, B.Vanderborght, and D. G. Caldwell, "A novel actuator with adjustable stiffness (AWAS)," International Conference on Intelligent Robots and Systems (IROS2010), 2010.

[5] B. Berret, S. Ivaldi, F. Nori, and G. Sandini, "Stochastic optimal control with variable impedance manipulators in presence of uncertainties and delayed feedback," in International Conference on Intelligent Robots and Systems (IROS2011). IEEE, 2011, pp. 4354-4359.

[6] A. W. Wiegner and R. L. Watts, "Elastic properties of muscles measured at the elbow in man: I. normal control," J Neurol Neurosurg Psychiatry, 1986.

[7] S. Koley and A. Singh, "Trends of active range of motion at three important joints in school-going boys of amritsar," Anthropologist, 2008.

[8] R. Beira, M. Lopes, M. Praça, J. Santos-Victor, A. Bernardino, G. Metta, F. Becchi, and R. J. Saltarén, "Design of the robot-cub (icub) head," in International Conference in Robotics and Automation (ICRA2006. IEEE, 2006, pp. 94-100.

[9] "Bll-blp datasheet," Istituto Italiano di Tecnologia, http://eris:liralab:it/wiki/Controller_cards.

[10] N. Schmit and M. Okada, "Synthesis of a non-circular cable spool to realize a nonlinear rotational spring," in International Conference on Intelligent Robots and Systems (IROS2011). IEEE, 2011, pp. 762767. 


\section{pnrVSA}

\section{Passive Noise Rejecting Variable Stiffness Actuator}

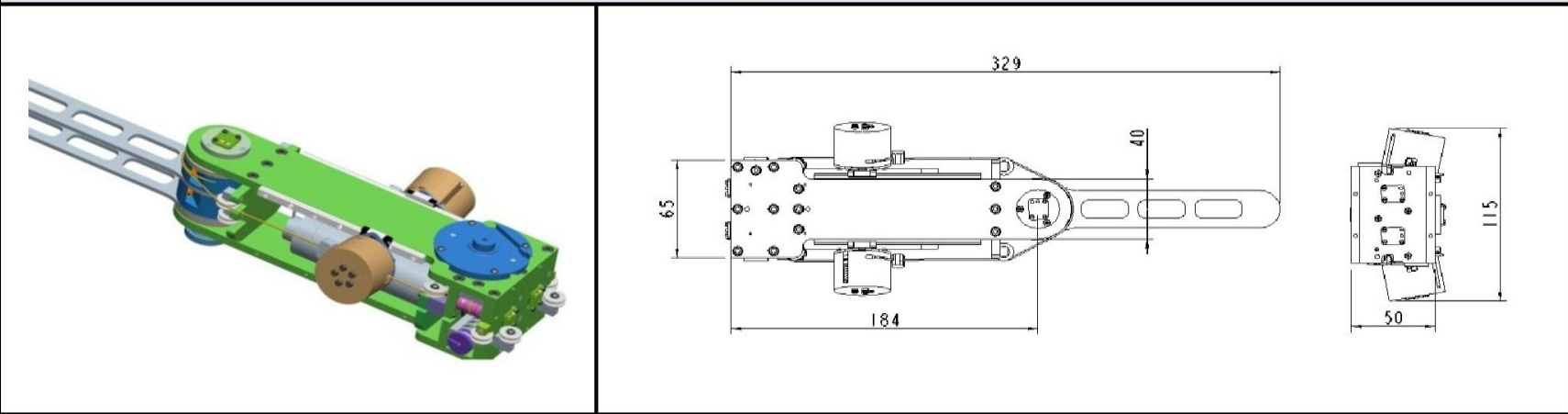

\begin{tabular}{|c|c|c|c|c|}
\hline \multicolumn{5}{|c|}{ Operating Data } \\
\hline$\#$ & \multicolumn{2}{|c|}{ (quantity) } & (unit) & (value) \\
\hline \multicolumn{5}{|c|}{ Mechanical } \\
\hline 1 & \multicolumn{2}{|c|}{ Continuous Output Power } & {$[\mathrm{W}]$} & 23 \\
\hline 2 & \multicolumn{2}{|c|}{ Nominal Torque } & {$[\mathrm{Nm}]$} & 1 \\
\hline 3 & \multicolumn{2}{|c|}{ Nominal Speed } & {$[\mathrm{rad} / \mathrm{s}]$} & 16,82 \\
\hline 4 & \multirow{2}{*}{$\begin{array}{c}\text { Nominal Stiffness } \\
\text { Variation Time }\end{array}$} & with no load & {$[\mathrm{s}]$} & 0,2 \\
\hline 5 & & with nominal torque & {$[\mathrm{s}]$} & 0,2 \\
\hline 6 & \multicolumn{2}{|c|}{ Peak (Maximum) Torque } & {$[\mathrm{Nm}]$} & 5,15 \\
\hline 7 & \multicolumn{2}{|c|}{ Maximum Speed } & {$[\mathrm{rad} / \mathrm{s}]$} & 17,94 \\
\hline 8 & \multicolumn{2}{|c|}{ Maximum Stiffness } & {$[\mathrm{Nm} / \mathrm{rad}]$} & 11,92 \\
\hline 9 & \multicolumn{2}{|c|}{ Minimum Stiffness } & {$[\mathrm{Nm} / \mathrm{rad}]$} & 0 \\
\hline 10 & \multicolumn{2}{|c|}{ Maximum Elastic Energy } & {$[\mathrm{J}]$} & 1,7 \\
\hline 12 & \multirow{2}{*}{ Maximum deflection } & with max stiffness & {$\left[{ }^{\circ}\right]$} & 60 \\
\hline 13 & & with min stiffness & {$\left[{ }^{\circ}\right]$} & 60 \\
\hline 14 & \multicolumn{2}{|c|}{ Active Rotation Angle } & {$\left[{ }^{\circ}\right]$} & $+/-120$ \\
\hline 15 & \multicolumn{2}{|c|}{ Angular Resolution } & {$\left[{ }^{\circ}\right]$} & 0,032 \\
\hline 16 & \multicolumn{2}{|c|}{ Weight } & [Kg] & 0.65 \\
\hline \multicolumn{5}{|c|}{ Electrical } \\
\hline 17 & \multicolumn{2}{|c|}{ Nominal Voltage } & [V] & 48 \\
\hline 18 & \multicolumn{2}{|c|}{ Nominal Current } & [A] & 1 \\
\hline 19 & \multicolumn{2}{|c|}{ Maximum Current } & [A] & 4 \\
\hline \multicolumn{5}{|c|}{ Control } \\
\hline 20 & \multicolumn{2}{|c|}{ Voltage Supply } & [V] & 48 \\
\hline 21 & \multicolumn{2}{|c|}{ Nominal Current } & {$[\mathrm{A}]$} & 0,06 \\
\hline 22 & \multicolumn{2}{|c|}{ I/O Protocol } & [] & CAN \\
\hline
\end{tabular}
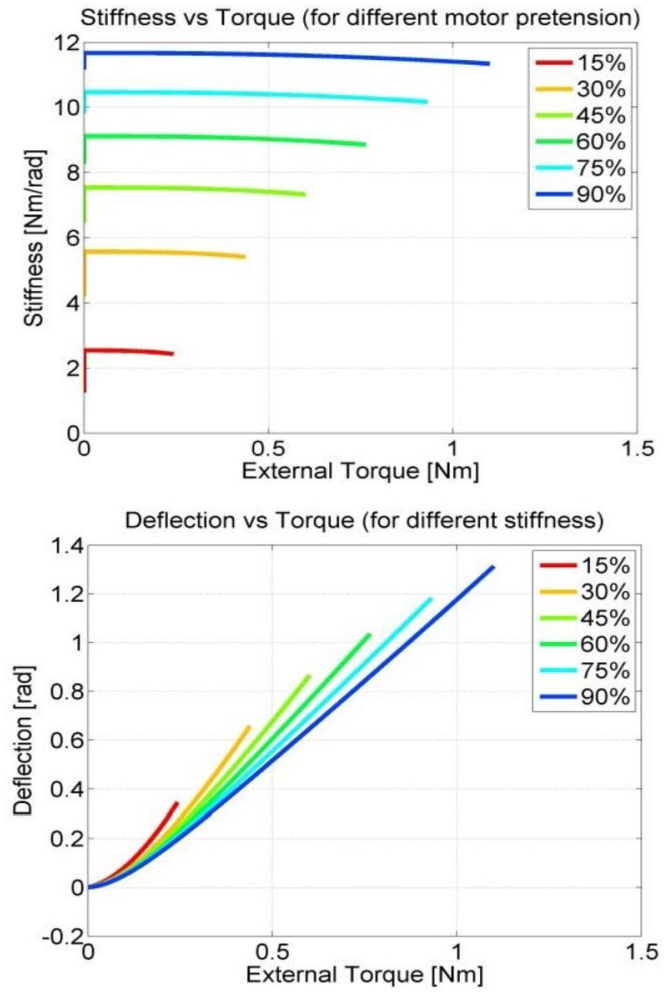

Actuator force vs Joint position (different motor pretension)

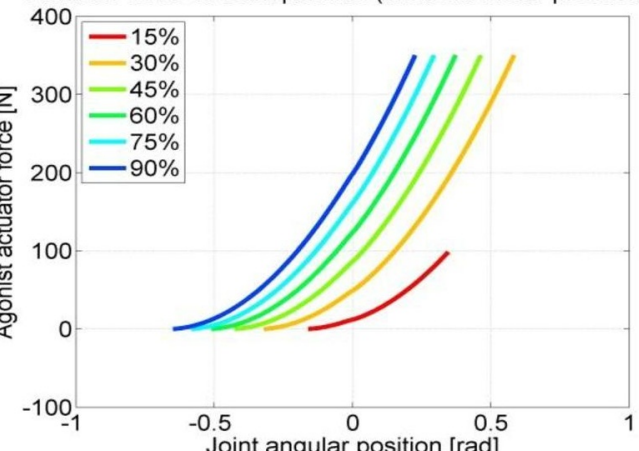

Fig. 10. The VIACTORS Variable Stiffness Joint Datasheet was developed within the VIACTORS project, which is a part of the EU 7th Framework Programme. It is intended to form a basis for the exchange of information of different VSJs on an objective basis. In the plots on the right hand side we report the nrpVSA characteristic curves for different internal motor pretensions. This pretension has to be interpreted as the applied torque at motor reels, ranging from 15 to 90 percent of the stall torque. The Datasheets of different Variable Stiffness Joints and the template can be downloaded from the VIACTORS homepage http://www.viactors.org/. 